\title{
¿Es necesaria una segunda reforma de la atención primaria en España?
}

\author{
Is a second reform of primary health care in Spain necessary?
}

Alberto Infante Campos ${ }^{1}$

Adriana Cavalcanti de Aguiar ${ }^{2}$

${ }^{1}$ Escuela Nacional de

Sanidad, Instituto de Salud Carlos III. Avda. Monforte de Lemos 5, Pab. VIII.

Madrid. ainfante@isciii.es.

${ }^{2}$ Mestrado Profissional em

Saúde da família,

Universidade Estácio de Sá.

\begin{abstract}
Created in 1986, the Spanish National Health System adopted Primary Health Care (PHC) as one of its building blocks. Since the beginning, the Spanish PHC model has been the subject of both praise and criticism. Nowadays, a crisis in public policy funding - including health funding policies - is resurrecting old debates and raising new ones. This paper analyzes the data available about the degree of acceptance of $\mathrm{PHC}$ from the perspective of Spanish citizens as well as data about the debate produced in the last five years by politicians, patients and health workers. At the end of the paper the authors seek to draw some conclusions based on the commonly acknowledged strengths and weakness of the PHC model, which decision-makers ought to take into consideration in order to avoid mistakes caused by improvised decisions elicited by the requirements of the moment.
\end{abstract}

Key words Health policies, Primary Health Care, Health indicators
Resumen El Sistema Nacional de Salud (SNS) español, creado en 1986, tiene como uno de sus pilares la atención primaria de salud (APS). Desde su nacimiento, el modelo español de APS ha sido objeto de elogios y de críticas. El actual momento de crisis de financiación de las politicas públicas, entre ellas la de salud, está resucitando viejos debates y planteando otros nuevos. El presente artículo examina los datos que se conocen sobre el grado de aceptación del modelo de APS por parte de los ciudadanos españoles, así como datos del debate de administraciones, pacientes y profesionales durante los últimos cinco años. Al final, los autores tratan de extraer algunas conclusiones que los decisores politicos deberían tener en cuenta para, partiendo de las fortalezas y debilidades comúnmente admitidas del modelo de APS, hacer frente a la difícil situación actual evitando errores derivados de decisiones improvisadas a causa de las urgencias del momento.

Palabras-clave Politica de salud, Atención Primaria en Salud, Indicadores de Salud 


\section{Introducción}

En España, tanto en los medios "profesionales" como en los de difusión "general" periódicamente aparecen opiniones sobre la necesidad de reformar el Sistema Nacional de Salud (SNS) y, en concreto, el modelo de atención primaria de salud (APS) ${ }^{1}$ establecido a partir de 1986 tras la aprobación de la Ley General de Sanidad. Durante mucho tiempo estos debates se basaron en opiniones más o menos cualificadas o en encuestas de escasa representatividad. Sin embargo, gracias al trabajo desarrollado durante los últimos años podemos contar, por vez primera, con información fiable y accesible sobre el funcionamiento de la APS desde el punto de vista de la ciudadanía española, además de registros de la opinión de distintos actores sobre ese funcionamiento, material que fue examinado y evaluado durante la I Conferencia Nacional de APS, en octubre de $2011^{2}$.

Aunque el SNS español es efectivo con costes más bajos que otros sistemas de salud europeos ${ }^{3}$, desde finales de 2010 y durante 2011 algunas autoridades sanitarias regionales (por ejemplo, la Comunidad de Madrid con el establecimiento del Área Sanitaria Única o Cataluña con el cierre y reorganización de centros y servicios) han introducido cambios en la APS que están generando inquietud entre pacientes y profesionales. $\mathrm{Mu}$ chos de estos cambios se presentan como una "respuesta inevitable" a las dificultades de financiación de los servicios públicos, en particular de los servicios de salud, que experimentan los gobiernos regionales. El ritmo de estos cambios parece haberse acelerado las elecciones regionales y locales de mediados de 2011 que produjeron un vuelco en el color político de estos gobiernos. Esta tendencia se ha reforzado tras el cambio producido a finales de 2011 en el gobierno de la nación. Y aunque puede haber quedado oscurecida tras las últimas y llamativas medidas de recorte del gasto en salud recientemente anunciadas, siguen estando presente.

El objetivo de este artículo es llamar la atención sobre aquellos aspectos sobre los que existe un mayor consenso en la necesidad de emprender acciones de reforma que podrían mejorar la eficiencia y la calidad del modelo de APS sin poner en riesgo sus logros.

\section{Material y Métodos}

Para obtener datos acerca de qué opinan los ciudadanos acerca de la APS en España nos basamos en el Barómetro Sanitario (BS) que es un amplio estudio de opinión que, desde 1995, realiza cada año el Ministerio de Sanidad, Política Social e Inclusión (MSPSI) mediante un convenio con el Centro de Investigaciones Sociológicas. Esta encuesta realiza 7.800 entrevistas domiciliares a hombres y mujeres con 18 años o más, en todas las Comunidades Autónomas (CCAA), en tres momentos (o submuestras), con la intención de reflejar la situación media del año, representativa a nivel nacional. El error de muestreo es de $\pm 1,2 \%$ y el nivel de confianza del 95,5\% ${ }^{4}$.

De modo accesorio reportamos opiniones de los profesionales y las administraciones sanitarias sobre la situación de la APS según fueron presentadas en algunos eventos oficiales y corporativos, cuyos informes son de dominio público. Las fuentes consultadas fueron publicaciones de divulgación, informes de foros profesionales, declaraciones a la prensa. Como criterio se buscaron declaraciones en el sentido de que: a) la APS debe ser mantenida en los moldes actuales, b) es necesario promover cambios menores, c) se deben emprender cambios amplios y profundos.

\section{Resultados}

El SNS goza de buena acogida por parte de la ciudadanía. La Tabla 1 enseña la serie histórica de opiniones de los ciudadanos encuestados pelo BS sobre el grado de satisfacción con funcionamiento del SNS y necesidades eventuales de cambios desde 2005.

Para el último año con datos disponibles, 2010 , siete de cada 10 ciudadanos $(73,9 \%)$ consideraron que el SNS funciona bastante bien o bien. Utilizando una escala de satisfacción de 1 a 10 (grado de máxima satisfacción), el nivel de satisfacción con el funcionamiento del SNS se califica con 6,57 puntos (2010), idéntica para hombres y mujeres, manteniendo una tendencia de subida en los últimos 5 años. En una cuestión introducida en 2010 aparece que la APS fue el componente del sistema mejor apreciado (en relación con la asistencia especializada, los servicios de urgencias y los ingresos hospitalario), con 7,1 puntos en media.

Más que uno $70 \%$ de los respondientes informaron haber acudido a la consulta de un 
médico de familia en los 12 meses anteriores a la encuesta, desde 2005 (Tabla 2).

Ante la opción de poder elegir, los resultados obtenidos a partir de 2008 muestran que los ciudadanos elegirían centros públicos para consultas en APS (Tabla 3)

La Tabla 4 presenta el nivel de satisfacción con la atención recibida en las consultas de los médicos de familia de la sanidad pública.
El nivel de satisfacción con la atención prestada por los médicos de familia de la sanidad pública es elevado: en 2010 el 86,2\% manifiesta que dicha atención fue muy buena o buena (el $24,7 \%$ de las personas estima que las consultas fueron mejor o mucho mejor de lo que esperaban, uno 67,5 las consideró más o menos igual a su expectativa, y a uno 7,3 la atención recibida fue peor o mucho peor de lo que esperaba que fue-

Tabla 1. Opiniones sobre el funcionamiento del SNS (2005-2010)

\begin{tabular}{|c|c|c|c|c|c|c|}
\hline & 2005 & 2006 & 2007 & 2008 & 2009 & 2010 \\
\hline $\begin{array}{l}\text { "Funciona bastante bien + funciona bien, pero } \\
\text { necesita cambios" }(\%)\end{array}$ & 67,7 & 69,7 & 67,4 & 68,1 & 69,2 & 73,9 \\
\hline $\begin{array}{l}\text { "Necesita cambios fundamentales + debe rehacerse por } \\
\text { completo" }(\%)\end{array}$ & 31,1 & 29,4 & 31,6 & 31,1 & 30 & 25,1 \\
\hline $\begin{array}{l}\text { Grado de satisfacción con el funcionamiento del SNS } \\
\text { (escala 1-10) }\end{array}$ & 6,1 & 6,23 & 6,27 & 6,29 & 6,35 & 6,57 \\
\hline
\end{tabular}

Fuente: BS

Tabla 2. Comparecencia a consulta de médico de familia en los últimos 12 meses.

\begin{tabular}{lrrrrrr}
\hline & $\mathbf{2 0 0 5}$ & $\mathbf{2 0 0 6}$ & $\mathbf{2 0 0 7}$ & $\mathbf{2 0 0 8}$ & $\mathbf{2 0 0 9}$ & $\mathbf{2 0 1 0}$ \\
\hline Sí & 73 & 71,2 & 72,3 & 75 & 74,3 & 71,5 \\
No & 26,4 & 28,3 & 27,5 & 24,7 & 25,2 & 28,3 \\
No se acuerda o no contestó & 0,5 & 0,5 & 0,3 & 0,3 & 0,5 & 0,3 \\
\hline
\end{tabular}

Fuente: BS

Tabla 3. Tipo de servicio de elección para consultas en APS (2005-2010).

\begin{tabular}{lllllllll}
\hline & Público & \multicolumn{3}{c}{ Privado } & \multicolumn{3}{c}{ Ambos } \\
\hline $\mathbf{2 0 0 8}$ & $\mathbf{2 0 0 9}$ & $\mathbf{2 0 1 0}$ & $\mathbf{2 0 0 8}$ & $\mathbf{2 0 0 9}$ & $\mathbf{2 0 1 0}$ & $\mathbf{2 0 0 8}$ & $\mathbf{2 0 0 9}$ & $\mathbf{2 0 1 0}$ \\
62,9 & 62,8 & 63,9 & 31 & 31,3 & 29,8 & 5,6 & 5,5 & 5,7 \\
\hline
\end{tabular}

Fuente: BS

Tabla 4. Calidad de la atención recibida en las consultas del médico de familia sanidad pública.

\begin{tabular}{lrrrrrr}
\hline & $\mathbf{2 0 0 5}$ & $\mathbf{2 0 0 6}$ & $\mathbf{2 0 0 7}$ & $\mathbf{2 0 0 8}$ & $\mathbf{2 0 0 9}$ & $\mathbf{2 0 1 0}$ \\
\hline Muy buena & 19,4 & 21,1 & - & 21,1 & 25,1 & 26,4 \\
Buena & 64,8 & 61,7 & - & 63,8 & 61 & 59,8 \\
Regular & 12,5 & 14 & - & 12,2 & 11 & 11,1 \\
Mala & 2,2 & 1,7 & - & 1,5 & 1,7 & 2 \\
Muy mala & 0,5 & 0,7 & - & 0,7 & 0,6 & 0,5 \\
No sabe/no contestó & 0,7 & 0,7 & - & 0,7 & 0,5 & 0,1
\end{tabular}

Fuente: BS [Por un error, en la edición 2007 no se realizó esta pregunta]. 
se). Se les pidió que valorasen aspectos o circunstancias relacionadas con las consultas en la APS, como presenta la Tabla 5.

La mayoría de los aspectos investigados son valorados positivamente, con destaque para la cercanía de los centros, la confianza y seguridad que transmite el médico, el trato recibido del personal sanitario. Peor valorados aparecieron el tiempo que se tarda en hacer las pruebas diagnósticas y el tiempo de espera hasta entrar en la consulta. El BS informa que en 2010, la cita se la dieron siempre o casi siempre el mismo día en que la pedían los ciudadanos al 42\% (la media de espera para los demás $58 \%$ fue de 3,6 días).

También los profesionales se han manifestado sobre la situación de la APS. Tras las movilizaciones convocadas por la "Plataforma Diez Minutos" en 2006, el llamado "Compromiso de Buitrago" sistematizó 16 puntos con amplio apoyo sindical y ciudadano, manifestado por 41 entidades, que convergen para solucionar algunos problemas puntuados por los resultados del Barómetro Sanitario. En estos se destacan: incrementar los presupuestos de la APS (hasta la media de los países europeos desarrollados); garantizar condiciones de trabajo dignas, adecuando los recursos materiales y de plantilla a las necesidades de salud en cada región; adecuar los sistemas informáticos a las características de la APS (asegurando la confidencialidad de los pa- cientes y el secreto profesional); ampliar acceso a la tecnología diagnóstica y terapéutica, mediante adopción de guías de práctica clínica basada en la evidencia científica; establecer programas de formación continuada dentro del horario laboral; impulsar la investigación en la APS; incorporar innovaciones al modelo existente de forma prudente; profesionalizar la gestión; mejorar la coordinación entre Servicios Sociales y el Sistema Sanitario; crear incentivos por logros en efectividad y resolución de las necesidades sanitarias, y fomentar la participación ciudadana en la toma de decisiones.

A continuación, el Consejo Interterritorial del SNS (CISNS) constituyó una Comisión para proponer medidas de mejora ${ }^{6}$, formada por expertos (designados por las sociedades científicas de APS, las CCAA, las asociaciones de pacientes y el propio Ministerio). Esta Comisión elaboró un "Marco Estratégico para la mejora de la Atención Primaria en España: 2007-2012” (“Estrategia AP-21") ${ }^{7}$, con 44 líneas de actuación en torno a tres objetivos clave: calidad, eficacia y continuidad asistencial.

Declaraciones de portavoces cualificados de los médicos que trabajan en este nivel de atención reunidos en la XVI Conferencia del Comité Ejecutivo de la WONCA en 2010 han destacado que hay que seguir impulsando la Estrategia AP21 y que la Medicina de Familia debe convertirse

Tabla 5. Valoración de aspectos de la asistencia sanitaria pública prestada en consultas de medicina de familia y pediatría (escala 1-10).

\begin{tabular}{|c|c|c|c|c|c|c|}
\hline & 2005 & 2006 & 2007 & 2008 & 2009 & 2010 \\
\hline La cercanía de los centros & 7,62 & 7,68 & 7,64 & 7,53 & 7,74 & 7,85 \\
\hline El trato recibido del personal sanitario & 7,38 & 7,36 & 7,33 & 7,35 & 7,42 & 7,50 \\
\hline El tiempo dedicado por el médico a cada enfermo & 6,49 & 6,49 & 6,32 & 6,4 & 6,58 & 6,76 \\
\hline $\begin{array}{l}\text { El conocimiento del historial y seguimiento de los } \\
\text { problemas de salud de cada usuario }\end{array}$ & 6,97 & 6,97 & 6,89 & 6,95 & 7,05 & 7,26 \\
\hline La facilidad para conseguir cita & 6,59 & 6,63 & 6,45 & 6,54 & 6,51 & 6,89 \\
\hline La confianza y seguridad que transmite el médico & 7,40 & 7,38 & 7,27 & 7,35 & 7,40 & 7,54 \\
\hline El tiempo de espera hasta entrar en consulta & 5,59 & 5,58 & 5,48 & 5,52 & 5,56 & 5,79 \\
\hline $\begin{array}{l}\text { Cuando lo necesita, el médico de cabecera le envía } \\
\text { a un especialista }\end{array}$ & 7,10 & 7,20 & 7,09 & 7,17 & 7,19 & 7,26 \\
\hline $\begin{array}{l}\text { El equipamiento y medios tecnológicos existentes } \\
\text { en los centros }\end{array}$ & 6,48 & 6,71 & 6,55 & 6,49 & 6,66 & 6,87 \\
\hline La información recibida sobre su problema de salud & 7,16 & 7,11 & 7,06 & 7,06 & 7,20 & 7,34 \\
\hline $\begin{array}{l}\text { El tiempo que tarda el médico en verle desde que } \\
\text { pide la cita }\end{array}$ & 6,57 & 6,44 & 6,32 & 6,22 & 6,18 & 6,44 \\
\hline $\begin{array}{l}\text { El tiempo que se tarda en hacer las pruebas } \\
\text { diagnósticas }\end{array}$ & - & - & 5,26 & 5,22 & 5,24 & 5,45 \\
\hline
\end{tabular}

Fuente: BS 
en asignatura de pregrado en todas las Facultades de Medicina ${ }^{8}$. Casi simultáneamente, representantes de importantes colectivos de APS $^{9}$ insistían en que se requiere mayor financiación, menos burocracia y más tiempo por paciente, y que se debe fomentar la investigación orientada a los problemas de la comunidad. Por su parte, los profesionales de enfermería han venido reclamando durante los últimos dos años la creación de la especialidad de enfermería de APS $^{10}$ así como la puesta en marcha del sistema de prescripción enfermera, lo que contribuiría para minimizar las esperas apuntadas por el Barómetro Sanitario.

Siguiendo la línea de pronunciamientos anteriores $^{11,12}$ en la reciente I Conferencia Nacional de APS, los representantes de los distintos colectivos de APS insistieron en que para mejorarla habría que aumentar el porcentaje del presupuesto sanitario público destinado a APS hasta los 25\%, dando a los profesionales un mayor acceso a pruebas diagnósticas; redistribuir mejor el tiempo para garantizar la formación y la investigación en la APS, fortaleciendo a la Medicina de Familia en la enseñanza de pregrado, además de seguir desarrollando y evaluando la Estrategia AP-21.

Políticos y administradores sanitarios de diferentes matices partidarios han optado por aproximaciones bastante coincidentes con las de la población y de la mayoría de las organizaciones profesionales, es decir: mantener los rasgos básicos del actual modelo de APS e irlo mejorando mediante cambios parciales y paulatinos. En 2010 la Ministra de Sanidad, señaló el interés de su departamento en "solventar el déficit de especialistas en Medicina Familiar y Comunitaria (MFyC) en el SNS" ampliando plazas de residencia médica; "desarrollar la troncalidad en la formación de las especialidades médicas gracias a la cual la Medicina Familiar y Comunitaria impregnará al resto de las disciplinas del tronco médico"; "fomentar la especialidad de enfermería familiar y comunitaria", y "impulsar la formación continuada y el desarrollo profesional”13. Algunos Consejeros de Sanidad ${ }^{14}$ de diferentes partidos que han gobernado las CCAA han insistido en: aumentar la capacidad resolutiva de la APS; mejorar la coordinación con la asistencia especializada; seguir disminuyendo el número de personas su la responsabilidad de cada médico de familia, y seguir impulsando la informatización de la asistencia por la vía de la Historia Clínica Digital y la receta electrónica.

\section{Discusión}

De todo lo anterior parece deducirse que existe un amplio consenso sobre las fortalezas del modelo de APS y, también, sobre algunas de sus debilidades. El modelo, en términos generales, ha funcionado bien y se ha mantenido bastante estable durante los últimos veinticinco años, con participación de la ciudadanía. En 2002, con la aprobación de la Ley Básica Reguladora de la autonomía del paciente y de derechos y obligaciones en materia de información y documentación clínica ${ }^{15}$, buena parte de las asociaciones de pacientes se han orientado a hacer cumplir los preceptos de dicha ley. Así, han ido obteniendo voz en el diseño y la evaluación de las estrategias de salud, como en el Foro Abierto establecido por el artículo 67 de la Ley de Cohesión y Calidad del SNS ${ }^{16}$ en 2003, estimulando "organizaciones, consejos, sociedades o asociaciones" para "el estudio, debate y formulación de propuestas sobre temas específicos que en un determinado momento tengan impacto en el Sistema Nacional de Salud."

Como en todo sistema dinámico, también en la APS se han ido produciendo cambios a lo largo de los últimos años, algunos de los cuales se reflejan en el primer informe de evaluación de la estrategia AP-21 presentado en el pleno del CISNS de febrero de $2010^{17}$. Además de la mayoritaria opinión positiva de los ciudadanos, este informe destaca: el porcentaje del gasto sanitario público en APS no ha subido entre 2002 y 2008, mientras el porcentaje del gasto en farmacia se redujo cuatro puntos porcentuales; el rango del porcentaje de gasto en APS entre las distintas CCAA varió entre el $11.2 \%$ y el $17.7 \%$ del gasto sanitario público; disminuyó paulatinamente el número de pacientes (o de Tarjetas Sanitarias Individuales) por profesional de la medicina y de la enfermería de APS; todas las CCAA usan guías de práctica clínica (GPC) como herramienta de apoyo a las decisiones asistenciales ${ }^{18}$, aunque se observa una notable variabilidad de criterios, por ejemplo en la indicación de pruebas diagnósticas; casi todas las CCAA han desarrollado acciones para reducir las cargas burocráticas en los centros de APS; la mayoría de CCAA ofrecieron acciones formativas volcadas para la calidad de la atención y la seguridad del paciente. Entre los aspectos mejorables se destacan la valoración relativa de la correcta comunicación entre niveles asistenciales; la demora (en días) entre que se pide la cita y se atiende a la persona; la dispensación electrónica de medicamentos. Una segunda evaluación deberá ser presentada al CISNS en 2012, buscando depurar parte 
de los indicadores cuantitativos y evaluar de forma más precisa la información aportada por las CCAA, de nuevo con la participación de las asociaciones de profesionales.

\section{Conclusiones}

Tanto los ciudadanos como los profesionales y administradores de diversos matices no demandan cambios radicales en el modelo de APS vigente en España, sino que más bien destacan determinadas áreas de mejora, que están bien identificadas desde hace tiempo. El análisis de los datos de Barómetro Sanitario aclara que los pacientes quieren esperar menos para obtener una cita, entrar en consulta y obtener una prueba diagnóstica, así como en un trato más personalizado. Por su vez, los profesionales de APS reclaman una mayor financiación, mayor continuidad de la atención, menos burocracia y más tiempo por paciente. Las administraciones se empeñan en aumentar la informatización de la gestión admi- nistrativa, del manejo de la información clínica y de las recetas médicas, lo que disminuiría el gasto con fármacos. Son también necesarias más investigaciones sobre las condiciones de organización y de ejercicio profesional en la APS (por ejemplo, sobre el papel de los distintos esquemas de incentivos al personal sanitario).

Las conclusiones y recomendaciones de la segunda evaluación de la Estrategia AP-21, deberían hacerse públicas tal como estaba previsto, y ser ampliamente debatidas y usadas para sustentar las decisiones políticas en el corto y en el mediano plazo. De ese modo se evitaría que la llegada de una gran número de nuevos responsables sanitarios a los centros de decisión unida a las fuertes presiones para reducir el gasto público lleve a la adopción de medidas más basadas en el corto plazo y en consideraciones financieras que en la experiencia acumulada y en las oportunidades detectadas para mejorar la eficiencia y la efectividad de la APS. Tal como recientemente han puesto de manifiesto las organizaciones profesionales de los médicos ${ }^{19}$.

\section{Colaboradores}

A Infante fue responsable por la concepción y el diseño del manuscrito, en la adquisición de los datos, en su análisis e interpretación, y redactó el manuscrito y aprobó la última versión. A Aguiar contribuyó en el diseño, participó en la adquisición de datos y su análisis y en la redacción del artículo, revisó el artículo críticamente en cuanto a contenido intelectual y aprobó la última versión.

\section{Agradecimientos}

Los autores agradecen a la Fundación Carolina, a la Universidade Estácio de Sá, a IMS-UERJ y a los funcionarios del Instituto de Información Sanitaria del Ministerio de Sanidad y Política Social (MSPS) y, en particular, a Mari Santos Ichaso y a Mercedes Alfaro la información facilitada y los comentarios aportados. 


\section{Referencias}

1. Peiró S, Artells J, Meneu R. Identificación y priorización de actuaciones de mejora de la eficiencia en el Sistema Nacional de Salud. Gac. Sanit 2011; 25: 95-105.

2. I Conferencia Nacional de APS, en octubre de 2011. [site na Internet]. [acessado 2012 nov 2]. Disponible en: http://www.msps.es/gabinetePrensa/notaPrensa/desarrolloNotaPrensa.jsp?id=2233

3. The Organisation for Economic Co-operation and Development (OECD). OECD Health Data 2011. [site na Internet]. [acessado 2012 nov 2]. Disponible en: www.oecd.org/health/healthdata

4. España. Ministerio de Sanidad, Servicios Sociales e Igualdad. Opinión de los ciudadanos. Barómetro Sanitario. [site na Internet]. [acessado 2012 nov 2].Disponible en: http://www.msps.es/estadEstudios/estadisticas/sisInfSanSNS/informeAnual.htm

5. Observatório sanitário en atención primária. El Compromiso de Buitrago. [site na Internet]. [acessado 2012 nov 2]. Disponible en: http://www. observatoriosanitario.org/ElCompromisodeBuitrago /tabid/70/Default.aspx

6. Martín M. Los planes autonómicos de mejora de la Atención primaria. En: Luis Palomo L, editor. Expectativas y Realidades de la Atención Primaria Española. Madrid: Fundación $1^{\circ}$ de Mayo y Ediciones GPS; 2010. 364 p.

7. España. Ministerio de Sanidad, Servicios Sociales e Igualdad. Proyecto AP21. Estrategias para la Atención Primaria del Siglo XXI. [site na Internet]. [acessado 2012 nov 2]. Disponible en: http://www.msps. es/profesionales/proyectosActividades/Proyecto AP21/home.htm

8. XVI Conferencia del Comité Ejecutivo de la WONCA, Málaga, Diario Médico, octubre de 2010.

9. XXXII Congreso de Semergen, Maspalomas, Diario Médico, octubre 2010.

10. Federación de Asociaciones de Enfermería Comunitaria y Atención Primaria. Manifiesto por la especialidad de Enfermería Familiar y Comunitaria. Madrid 2009

11. Declaración de Málaga. Global Family Doctor 36, 2010 [site na Internet]. [acessado 2012 nov 2]. Disponible en: http://www.globalfamilydoctor.com/site/ DefaultSite/filesystem/documents/newsArchive/201012.pdf
12. XXXII Congreso de Sociedad Española de Médicos de Atención primaria (Semergen). [site na Internet]. [acessado 2012 nov 2]. Disponible en: http:// www.semergen.es/semergen/congresos-y-jornadasnacionales

13. Ministra de Sanidad y Política Social. Intervención en el XXXII Congreso de Semergen. Maspalomas, octubre de 2010.

14. Consejeros de Sanidad de Castilla y León y Canarias en XXXII Congreso de Semergen. Maspalomas, octubre de 2010. [site na Internet]. [acessado 2012 nov 2]. Disponible en: http://www.mspsi. gob.es/gabinetePrensa/notaPrensa/desarrollo NotaPrensa.jsp?id=1732

15. España. Ley $41 / 2002$, de 14 de noviembre, básica reguladora de la autonomía del paciente y de derechos y obligaciones en materia de información y documentación clínica. Disponible en: http:// www.boe.es/boe/dias/2002/11/15/pdfs/A4012640132.pdf

16. España. Ley 16/2003 de 28 de mayo, de Cohesión y Calidad del Sistema Nacional de Salud. Disponible en: http://www.boe.es/aeboe/consultas/bases_datos /doc.php?id=BOE-A-2003-10715

17. Consejo Interterritorial del Sistema Nacional de Salud. Acciones y medidas para promover la calidad, la equidad, la cohesión y la sostenibilidad del SNS. Madrid 18 de marzo de 2010. [site na Internet]. [acessado 2012 nov 2]. Disponible en: http://www. mpdc.es/components/com_mtree/attachment. php?link_id=466\&cf_id=39

18. Portal Guía-Salud del SNS. [site na Internet]. [acessado 2012 nov 2]. Disponible en: http://www. guiasalud.es/web/guest/gpc-sns

19. Organización Médica Colegial. Comunicado de la Asamblea General. Madrid 28 de enero de 2012.

Artigo apresentado em 25/03/2012

Aprovado em 10/04/2012

Versão final apresentada em 16/05/2012 